\title{
More data to encourage current cigarette smokers to quit
}

A link between smoking and coronary heart disease was noted $>50$ years ago, and much evidence for a causal role for smoking has accrued since then. A large meta-analysis of individual data from 25 cohort studies has now been performed to assess the association between cigarette smoking and cardiovascular disease in what the investigators describe as a "previously understudied population"adults aged $\geq 60$ years.

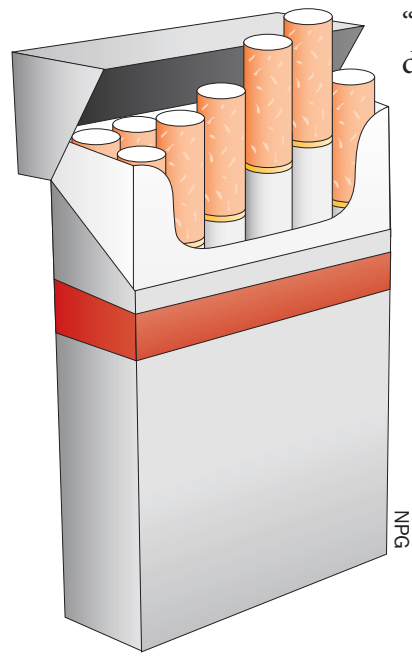

"Given current demographic trends, prevention in older adults through risk factor management is of crucial importance to reduce the burden of cardiovascular disease," say the investigators.

The research team focused their 긍 analysis on the 503,905 cohort study participants aged $\geq 60$ years. At baseline, $40.2 \%$ of these individuals had reported that they were 'never smokers' (never having smoked daily or regularly, or having smoked $\leq 100$ cigarettes over their lifetime), $47.4 \%$ said they were former smokers, and $12.4 \%$ were classified as current smokers. The primary outcome of the study was death from a cardiovascular cause; 37,952 such deaths were recorded during follow-up, which ranged from 1.6 years to 15.4 years.

Participants classified as current smokers at baseline were twice as likely to have died from a cardiovascular cause by follow-up (HR 2.07, 95\% CI 1.82-2.36), and were estimated to die from a cardiovascular cause 5.50 years earlier than 'never smokers'. Among the current smokers, the increased risk of cardiovascular death was found to be higher with greater cigarette consumption; for every 10 cigarettes smoked each day, the hazard ratio for death from a cardiovascular cause increased by 1.40 .

Individuals classified as former smokers at baseline were also more likely to have died from cardiovascular causes by follow-up (HR 1.37, 95\% CI 1.25-1.49), and were estimated to die from a cardiovascular cause 2.16 years earlier than 'never smokers'. Notably, however, the increased risk of cardiovascular death was found to diminish with time since the individuals had quit smoking; for every 10 years since smoking cessation, the hazard ratio for death from a cardiovascular cause decreased by 0.85 .

The investigators highlight that "studies suggest that older smokers are less likely to receive smoking cessation support than younger smokers". They point out that "in contrast to common misperceptions that older adult smokers might be too old to benefit from quitting, our findings indicate that smoking cessation should be encouraged at any age".

\section{Bryony M. Mearns}

Original article Mons, U. et al. Impact of smoking and smoking cessation on cardiovascular events and mortality among older adults: meta-analysis of individual participant data from prospective cohort studies of the CHANCES consortium. BMJ 350, h1551 (2015) 